\title{
R5 Plataforma alternativa e flexível para o Kit NAT brasileiro
}

Antonio G. P. Ferreira1', Elisabete Andrade'1, Daniele Rocha', Marcela Fontana $^{1}$, Marisa Ribero ${ }^{1}$, Elaine Motta ${ }^{1}$, Patrícia Alvarez ${ }^{1}$

${ }^{1}$ Bio-Manguinhos, Fiocruz, Rio de Janeiro, RJ

Introdução: O kit NAT HIV/HCV Brasileiro produzido por BioManguinhos foi desenvolvido para atender altas capacidades de processamento. A abertura de pool de amostras e o processamento de pequenas rotinas significam um considerável custo no processamento no formato atual do NAT. O estabelecimento de uma Plataforma NAT de equipamentos focado no processamento de poucas amostras 1230 reações, permitirá um melhor aproveitamento das rotinas e economia de recursos, permitindo 0 atendimento de potenciais serviços com pequenas demandas que estejam alinhadas com a estratégia de descentralização de ensaios NAT, na Hemorede Brasileira.

Objetivo: O objetivo do Flex NAT é definir e padronizar um modelo alternativo de produto/Kit NAT, processado numa plataforma de equipamentos flexível, contemplando os requisitos técnicos e operacionais definidos pela Hemorede Brasileira, com custo viável. Com um considerável nível de nacionalização dos insumos e, se possível, agregando processos de TT. Esta proposta de novo produto, baseia-se em um ensaio discriminatório, independente, com sensibilidade, especificidade, reprodutibilidade e rastreabilidade, comparáveis com o Kit NAT HIV/HCV já desenvolvido por BioManguinhos.

Metodologia: Foram identificadas alternativas de sistemas de extração de ácidos nucléicos, equipamentos/ insumos, baseados em beads magnéticos: Prepito/Chemagen (Perkin Elmer), EZ1 (QIAGEN) e Nimbus/ Biopur (Biometrix). A comparação desses sistemas de extração teve como parâmetro o MDx (QIAGEN), equipamento da plataforma NAT. Visando melhor sensibilidade/especificidade/reprodutibilidade quando comparado com o equipamento MDx os protocolos de extração de ácidos nucléicos foram ajustados. Os ajustes realizados incluíram: alterações dos volumes de amostra, eluição, partícula magnética, concentração de protease, tempos de lise e de secagem das beads magnéticas, entre outros. Foram utilizadas amostras HIV e HCV verdadeiras positivas, com cargas virais conhecidas.

Resultados: Os resultados, após comparação com MDx, demostraram que 0 equipamento Nimbus/Biopur, apresentou ganho de sensibilidade, cerca de $2 \mathrm{Cts}$, para amostras HIV/HCV na concentração de 500 cópias $/ \mathrm{mL}$ ou $\mathrm{UI} / \mathrm{mL}$, respectivamente. O equipamento EZ1 apresentou resultados de sensibilidade pouco satisfatórios. O 
Prepito/Chemagen, apresentou resultados bastante satisfatórios, com melhor sensibilidade e desempenho, com amostras HIV/HCV na concentração de até 12,5 cópias $/ \mathrm{mL}$ ou $25 \mathrm{Ul} / \mathrm{mL}$, respectivamente, sendo capaz de detectar 8 replicatas destas concentrações.

Conclusão: Os resultados preliminares demostram que os sistemas de equipamentos/insumos Prepito/Chemagen e Nimbus/ Biopur são promissores e altamente satisfatório para avançar na definição do modelo Flex NAT. Visando estabelecer este novo produto, será necessário estabelecer os níveis de sensibilidade, especificidade e reprodutibilidade dos kits/equipamentos. A padronização de um ensaio FLEX NAT e a possibilidade de aplicação nas rotinas de triagem de doadores de sangue no Brasil poderão gerar significativos impactos operacionais e de custo. Além disto, representa mais um exemplo de produto inovador para o Complexo Industrial da Saúde, contribuindo para consolidar competências tecnológicas na área de imunobiológicos e diagnóstico molecular na FIOCRUZ, atendendo à demanda de produtos estratégicos do Ministério da Saúde para o SUS.

Palavras-Chave: NAT, Kit, Flexivel 